\title{
SCIRROTHERIUM ANTELUCANUS,UNA NUEVA ESPECIE DE PAMPATHERIIDAE (MAMMALIA, XENARTHRA, CINGULATA) DEL MIOCENO SUPERIOR DE COSTA RICA, AMÉRICA CENTRAL
}

\author{
SCIRROTHERIUM ANTELUCANUS, A NEW SPECIES OF PAMPATHERIIDAE \\ (MAMMALIA, XENARTHRA, CINGULATA) FROM THE UPPER MIOCENE OF \\ COSTA RICA, CENTRAL AMERICA
}

\author{
César A. Laurito*1\&2 \& Ana L. Valerio ${ }^{3}$ \\ ${ }^{1}$ INA, Instituto Nacional de Aprendizaje \\ ${ }^{2}$ Investigador Asociado-Departamento de Historia Natural, \\ Museo Nacional de Costa Rica \\ ${ }^{3}$ Departamento de Historia Natural, Museo Nacional de Costa Rica. Apdo. \\ 749-1000, San José \\ *Autor para contacto: cesarlaurito@ice.co.cr
}

(Recibido: 01/02/2013 ; aceptado: 26/11/2013)

\begin{abstract}
For the first time the genus Scirrotherium is recorded and a new species is described for the early Hemphillian of Costa Rica. This finding constitutes a new clue about the early arrived of South American heralds to Central American during Pre-Great American Biotic Interchange, increasing by at least 3 Ma the record of the Pampatheriidae family in the Northern Hemisphere. The new Central America species described herein differs from the South American species of Scirrotherium because they have osteoderms with an inconspicuous ornamentation, a plain central figure and a remarkable area size.Keywords: Mammalia, Xenarthra, Pampatheriidae, Scirrotherium, Upper Miocene, Costa Rica, pre-GABI.
\end{abstract}

RESUMEN: Se registra por primera vez el género Scirrotherium y se describe una nueva especie para el Hemphilliano temprano de Costa Rica. Este hallazgo constituye una nueva evidencia sobre el arribo temprano de los heraldos sudamericanos a América Central durante el Pre-Gran Intercambio Biótico Americano, incrementando en al menos 3 Ma el registro de la familia Pampatheriidae en el Hemisferio Norte. La nueva especie de América Central difiere de las especies suramericanas de Scirrotherium por tener osteodermos con una ornamentación inconspicua, figura central simple y una muy amplia área superficial.

Palabras clave: Mammalia, Xenarthra, Pampatheriidae, Scirrotherium, Mioceno Superior, Costa Rica, pre-GABI. 


\section{INTRODUCCIÓN}

El cuerpo de los Pampatheriidae recuerda al de los verdaderos armadillos y comparte con los Dasypodidae y los Glyptodontidae el poseer una armadura bien desarrollada, constituida por numerosos osteodermos que se extiende sobre su cabeza, tronco, cola y miembros locomotores. Al contrario de los gliptodontes cuyo caparazón constituía un domo sólidamente suturado (Gillette \& Ray, 1981), el caparazón dorsal de los dasipódidos y pampatéridos es flexible debido a la presencia de bandas móviles en su parte media, constituidas por osteodermos especialmente modificados para traslaparse y articularse entre sí (Edmund, 1985), y a una no rígida unión entre los osteodermos. Los osteodermos son los elementos óseos más frecuentemente hallados en los yacimientos fosilíferos, su ornamentación ha sido utilizada por los científicos con cierto grado de confiabilidad en la realización de determinaciones taxonómicas, ello en ausencia de otros restos esqueletales (Edmund, 1985; Scillato-Yané et al., 2005), siendo este el caso de los pampatéridos.

De todos los Pampatheriidae el género Scirrotherium es el menos conocido y el menos frecuente; siendo $S$. hondaensis del Mioceno Medio de La Venta en Colombia, la especie más antigua conocida (Scillato-Yané et al., 2005), desde que la pertenencia del género Machlydotherium Ameghino, 1902 de edad Eoceno Tardío a esta familia fuera cuestionada (Hoffstetter, 1956; Carlini \& Scillato-Yané, 1993).

Por otra parte, la escasez de nuevos registros hizo pensar a los paleontólogos por cerca de dos décadas, de que se trataba de un género endémico y monoespecífico del noroeste de América del Sur. Sin embargo, recientemente se ha descrito una nueva especie de Scirrotherium fundamentada en osteodermos procedentes de las provincias de Chubut y Entre Ríos en Argentina (Cione et al., 2000, 2005; Dozo et al., 2002, 2008; Goís \& Scillato-Yané, 2010), y del Estado de Acre al noroeste de Brasil (Góis et al., 2013). Además, se han registrado restos de dicho género en Venezuela (Rincón, com. esc., 2009) y en el sur de América Central que están cambiando nuestra comprensión acerca de la paleobiogeografía, evo- lución y biocronología del género; permitiendo establecer nuevos elementos diagnósticos y una taxonomía más precisa y realista.

En América Central se han registrado hasta el momento 3 géneros de Pampatheriidae a saber: Pampatherium sp. en la localidad de Santa Amelia en Guatemala de edad Pleistoceno tardío (Woodburne, 1969); Holmesina con la especie $H$. septentrionalis en la localidad de Tomayate en El Salvador y de edad Blancano tardío-Irvingtoniano (Aguilar \& Laurito, 2009), y Scirrotherium sp. procedente de la Formación Curré del Mioceno Superior de Costa Rica (Laurito \& Valerio, 2008, 2010, 2012a).

De la Formación Curré, en años recientes, hemos colectado una importante muestra de osteodermos que ahora forman parte de la Colección de Fósiles de la Sección de Geología del Museo Nacional de Costa Rica y son el objeto de estudio del presente trabajo. Este material, corresponde a una nueva especie de Scirrotherium y fue incorrectamente citado de manera preliminar como perteneciente a los géneros Pampatherium y/u Holmesina por Mead et al. (2006). Dichos osteodermos constituyen parte de la evidencia más temprana del intercambio faunístico entre las Américas vía América Central meridional.

El material analizado, procede de la localidad fosilífera de San Gerardo de Limoncito, ubicada 14 $\mathrm{km}$ al oeste de la ciudad de San Vito en el cantón de Coto Brus, provincia de Puntarenas en las coordenadas 851'19.6" N y 8304'51.9" W (Fig.1), cuya edad ha sido asignada Hemphilliano Temprano tardío (Mioceno Tardío) con base en la fauna de caballos fósiles con los que se halló asociados (Valerio, 2010; Laurito \& Valerio, 2010, 2012a).

\section{METODOLOGÍA}

La identificación de la especie se fundamenta en el análisis de las similitudes y diferencias fenéticas de los osteodermos por comparación con otros pampatéridos siguiendo los criterios dados por Castellanos (1927, 1937, 1946), Edmund (1985, 1987, 1996); Edmund \& Theodor (1997); Góis et al. (2013) y otros autores (Figs. 2, 3 y 4). 

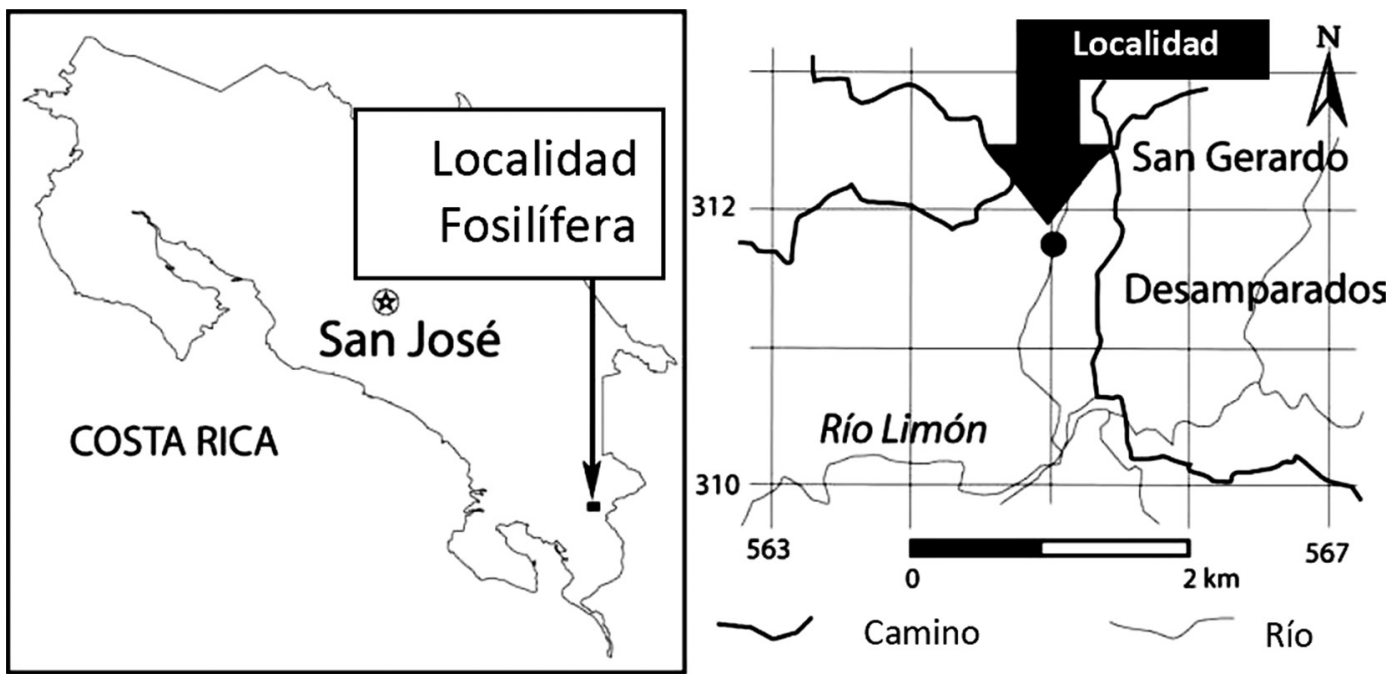

Fig. 1: Mapa de ubicación de la localidad fosilífera de San Gerardo de Limoncito, cantón de Coto Brus, provincia de Puntarenas.

También se aplica el análisis bivariado de las relaciones entre el área media y el espesor de los osteodermos fijos no marginales del caparazón, lo que permite de manera bastante confiable diferenciar las especies de Pampatheriidae del Mioceno, Plioceno y Cuaternario de Sudamérica y Norte América.

\section{PALEONTOLOGÍA SISTEMÁTICA}

MAMMALIA Linnaeus, 1758

XENARTHRA Cope, 1889

CINGULATA Illiger, 1811

GLYPTODONTOIDEA Gray, 1869

PAMPATHERIIDAE Paula Couto, 1954

SCIRrotherium Edmund \& Theodor, 1997

De acuerdo a Edmund \& Theodor (1997) el carácter más notable del género Scirrotherium está en la superficie ornamentada de los osteodermos no marginales, se trata de una única hilera de forámenes foliculares ubicada en el extremo anterior. Estos son relativamente grandes, escasos y están conectados entre sí por un canal distintivo. En principio este carácter permitiría diferenciar el género Scirrotherium de todos los restantes géneros de la Familia Pampatheriidae, pero como se discute más adelante este carácter no es suficiente.

\section{Scirrotherium antelucanus specie nova}

Etimología: del latín antelucanus o ante lucem que significa madrugador, anterior a la luz o al alba, en referencia al hecho de que es uno de los xenartros más antiguos hallado en América Central y es uno de los primeros mamíferos emigrantes de América del Sur en alcanzar el Arco de Islas Costa Rica-Panamá. Además, el hallazgo arroja luz sobre la época previa al desarrollo del Gran Intercambio Biótico entre las Américas.

Holotipo: CFM-2867 (fig. 6c).

Paratipos: CFM-1849 (fig. 6j), CFM-3543 (fig. 61), CFM-3546 (fig. 6q) y CFM-2559 (fig. $5 b)$.

Diagnosis de la especie: osteodermos fijos no marginales con dimensiones superiores a los de Scirrotherium hondaensis, inferiores a los de Vassallia máxima, superficies ligeramente mayores y anchos menores que Kraglievichia paranense (Figs. 2 y 4 ).

Osteodermos fijos no marginales de contorno hexagonal a cuadrangular con superficies dorsales bastante lisas, y amplios márgenes anteriores de 3 a $4 \mathrm{~mm}$ de ancho. El margen anterior contiene de 8 a 11 forámenes anteriores en los osteodermos hexagonales y 7 a 10 forámenes en los osteodermos cuadrangulares, con diámetros generales variables entre los 2 a $3 \mathrm{~mm}$. 


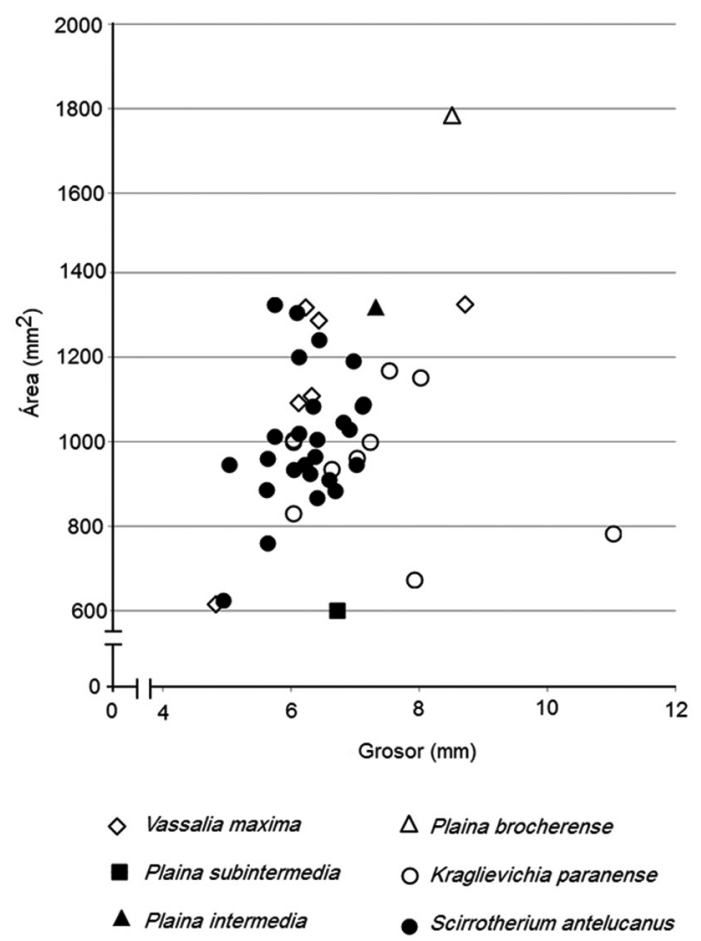

Fig. 2: Gráfica bivariable del grosor versus el área de osteodermos fijos no marginales de los géneros suramericanos de Pampatheriidae: Vassallia, Plaina y Kraglievichia comparados con Scirrotherium antelucanus, modificado de Rincón (com. esc., 2009).

Elevación marginal notablemente ancha, particularmente gruesas en los osteodermos hexagonales y depresiones longitudinales angostas y poco profundas.

La figura central o elevación central longitudinal elongada y delgada en los osteodermos cuadrangulares e inflada y ancha en los osteodermos hexagonales.

Osteodermos móviles con superficies articulares anteriores cortas; porciones intermedias ornamentadas que son ligeramente menos anchas que las superficies articulares, estas presentan de 1 a 3 filas de forámenes, cuando hay 2 o 3 hileras estos se disponen de manera alterna o incluso de manera desordenada. Las porciones posteriores son relativamente muy largas con el margen posterior igual o mucho más ancho que el margen anterior. Las elevaciones centrales varían de muy amplias a angostas y en general son poco elevadas y las depresiones longitudinales son muy angostas en contraposición las elevaciones marginales son muy anchas, limitadas por márgenes laterales que varían de angostos a prácticamente nulos.

Los osteodermos semi-móviles se caracterizan por porciones de las superficies articulares anteriores muy angostas, margen posterior con una pequeña protuberancia a manera de ápice, elevación central longitudinal corta, amplios márgenes laterales y angostas depresiones longitudinales (fig. 5h).

\section{Descripción}

El caparazón de los pampaterios está constituido por tres secciones, el escudo anterior o escapular y el escudo posterior o pélvico, separados por un cinturón flexible constituido por tres filas de osteodermos móviles imbricados (Edmund, 1985). Para efectos prácticos, no se pueden diferenciar los osteodermos de los escudos escapular y pélvico al menos que se recupere una buena parte articulada de los correspondientes escudos; pero si se tiene en cuenta la morfología conocida de otros pampaterios es posible lograr una adecuada asignación. Sin embargo, sí se puede diferenciar entre osteodermos fijos no marginales de los marginales y de los osteodermos de las bandas móviles; así como los osteodermos de los escudos cefálicos, de los miembros locomotores y los caudales.

A continuación se describen los diferentes tipos de osteodermos:

Osteodermos fijos (figs. 5a-o): los no marginales presentan contornos hexagonales a cuadrangulares con 5 o 6 lados. Presentan una única hilera de notables perforaciones en el borde marginal anterior, un sulcus poco profundo y amplio, y una figura central piriforme y angosta.

CFM-1818, osteodermo marginal (fig. 5m) de pequeño tamaño, presumiblemente de la porción nucal caracterizado por tener un contorno trapezoidal con una conspicua banda marginal anterior que presenta dos hileras de forámenes pilosos; su porción central está erodada y no se logra apreciar en detalle la figura central o eleva- 


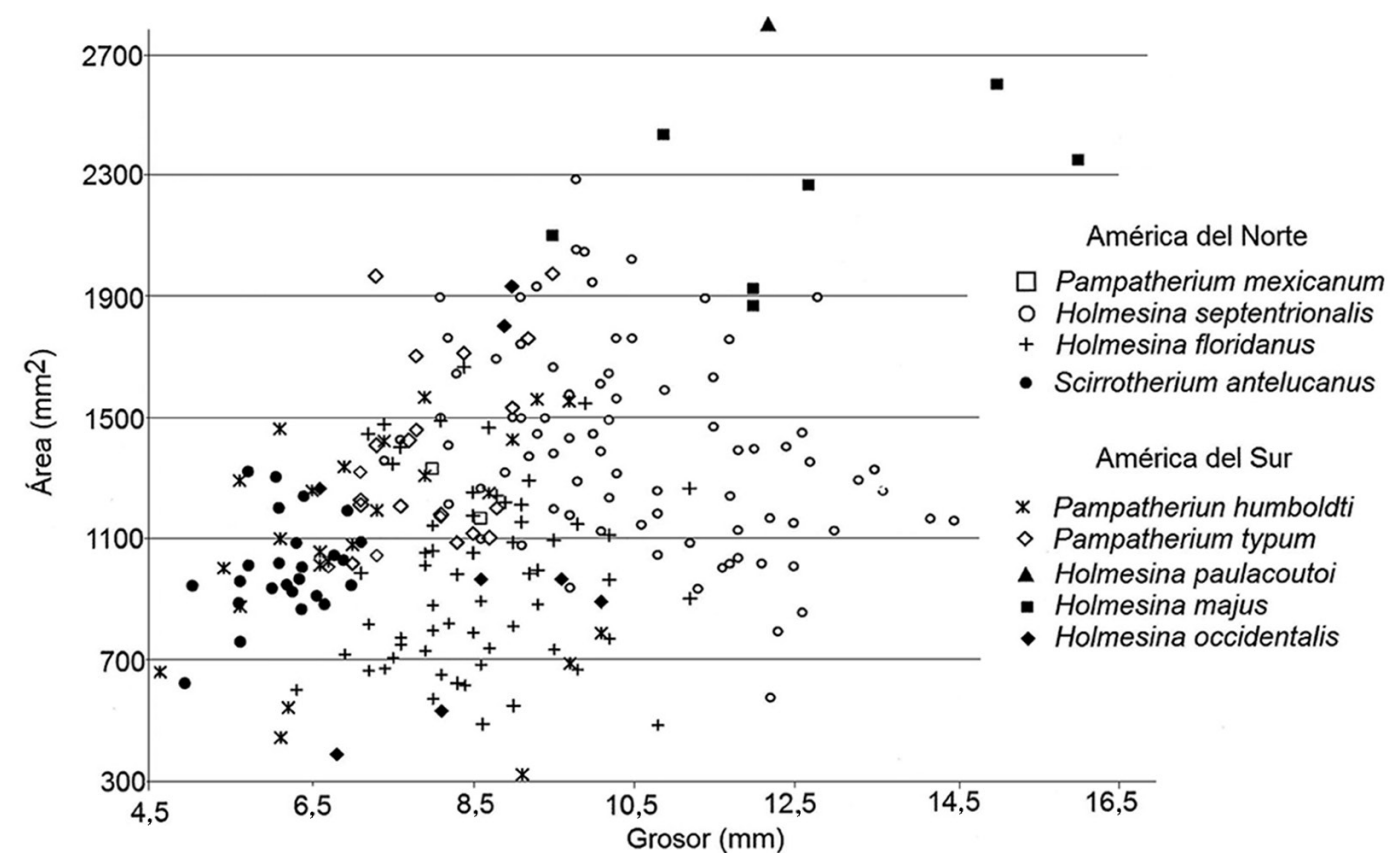

Fig. 3: Gráfica bivariable del grosor versus el área de osteodermos fijos no marginales de diferentes especies de Pampatheriidae de América, fundamentados en los trabajos de Rincón et al., (2008), Hulbert \& Morgan, (1993), Edmund, (1996) y datos adicionales compilados, graficados y suministrados por el Dr. Rincón (com. esc., 2009). Ver el detalle los datos biométricos de Scirrotherium antelucanus en el Apéndice.

ción central longitudinal; la elevación marginal es ancha y el sulcus aparentemente es amplio.

Osteodermos móviles (figs. 6a-j): los no marginales presentan un contorno rectangular; elevaciones centrales longitudinales, levemente elevadas, unidas a la elevación marginal anterior, rodeadas por un sulcus o depresión longitudinal angosta. Los osteodermos marginales presentan contornos que recuerdan un trapezoide más o menos simétrico con elevaciones centrales longitudinales bajas y muy anchas; en algunos ejemplares la elevación central longitudinal es muy ancha, abarcando casi toda la superficie externa del osteodermo, no pudiéndose distinguir el sulcus o depresión longitudinal ni la elevación marginal, pero en todos los casos si se observa un angosto margen lateral que está limitado en su margen anterior por una hilera de conspicuos forámenes en número variable de entre 6 a 9. La línea de forámenes está separada de la superficie articular anterior, por una banda transversal rugosa con abundantes poros en toda su superficie. La cara interna de los osteodermos es

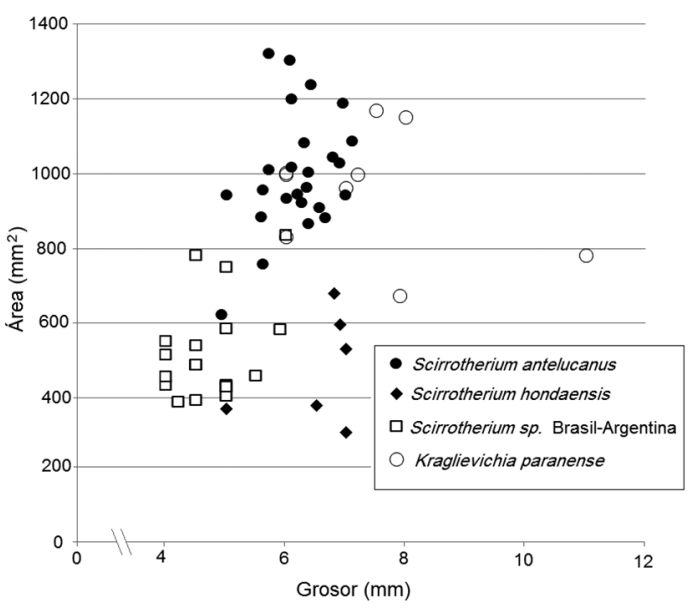

Fig. 4: Gráfica bivariable del grosor versus el área de osteodermos fijos no marginales de las diferentes especies de Scirrotherium descritas hasta ahora, comparadas con Kraglievichia paranense (apéndice 2). El rango de distribución de la especie Scirrotherium antelucanus, evidencia el mayor tamaño del área de sus osteodermos y un ancho menor al de Kraglievichia. Scirrotherium sp. de Brasil y Argentina, corresponde a Scirrotherium carinatum Góis et. al., 2013. 

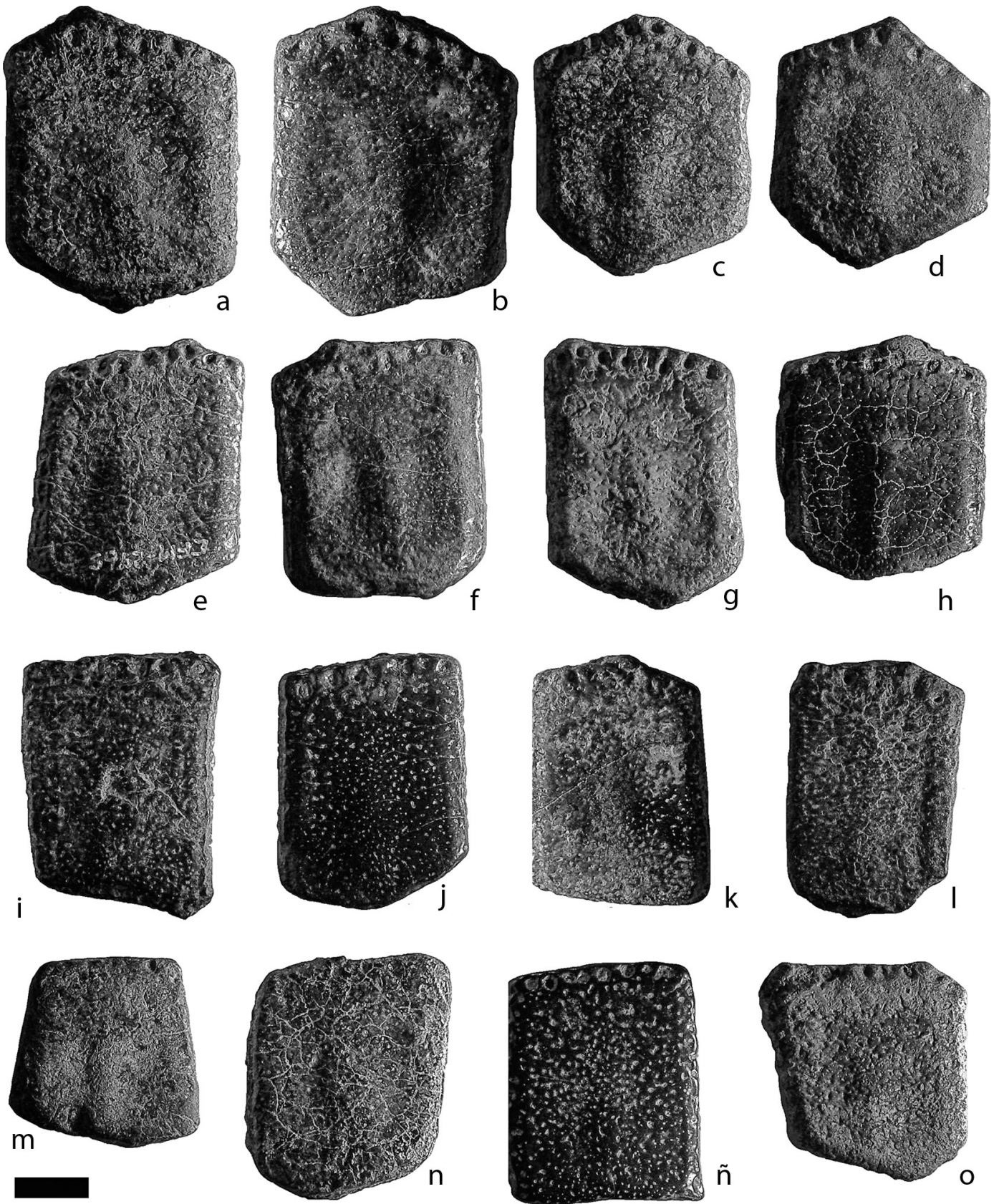

Fig. 5: a-o osteodermos fijos de las cinturas pélvica y/o escapular (escala gráfica $1 \mathrm{~cm}$ ).

cóncava en sentido tanto transversal, lisa con escasos poros vasculares.

En el caso del ejemplar CFM-1849 (fig. 6j), este pequeño osteodermo móvil, trapezoidal, presenta 3 filas de forámenes anteriores dispuestos de manera desordenada, separada de la superficie articular anterior por una porción intermedia rugosa relativamente amplia.

Osteodermos del escudo cefálico (figs. 6kp): presentan un contorno hexagonal, pentagonal 
o cuadrangular con elevación central longitudinal muy baja y amplia, apenas insinuada, con un sulcus muy somero, apenas visible y elevación marginal ancha; tienen un margen lateral angosto bien diferenciado con alrededor de 10 a 12 grandes forámenes entre anteriores y laterales que abarcan dos o tres lados del osteodermo. En algunos casos no es posible diferenciar la figura central, el sulcus o la elevación marginal, pero sí los márgenes laterales.

Osteodermos caudales (figs. 6q y 6r): se recuperaron dos osteodermos rectangulares alargados, longitudinalmente convexos, con el extremo posterior redondeado (ver figs. 24a-c de Castellanos, 1946 y fig. 12 de Edmund, 1985); en el ejemplar mejor preservado se observa una muy delgada y alargada elevación central longitudinal y márgenes laterales angostos bien diferenciados.

\section{Elementos de post-cráneo}

Se recuperaron 2 metatarsos completos y bien preservados. El CFM-2640 (figs. 7a-e) es un posible metatarso II izquierdo, con la vista plantar plana, mientras que la dorsal es ligeramente cóncava en sentido transversal, la faceta articular proximal es cóncava y la distal con una quilla media parcialmente erosionada del lado dorsal. Por su parte, el ejemplar CFM-3295 (figs. 7a'-e') corresponde a un posible metatarso III derecho, plano en su parte plantar y ligeramente cóncavo en la vista dorsal y en sentido transversal, con la faceta articular proximal inclinada hacia la parte plantar y una quilla media en la faceta articular distal del lado dorsal.

Dos fragmentos distales de fémur, el derecho (CFM-1639, figs. 8a-e) y el izquierdo (CFM3555, figs. 8a'-e'); ambos ensanchados en sentido lateromedial con el cóndilo lateral ligeramente más pequeño que el cóndilo medial. El tubérculo de la tróclea está parcialmente roto del lado lateral, en el caso del CFM- 3555 posiblemente causado por el ataque de un Crocodylidae ya que estos se encontraron asociados a gran cantidad de restos de cocodrilos. La cara poplítea es plana con una rugosidad en el centro de la misma y limitada por las tuberosidades supracondíleas, en el caso del CFM-1639 las tuberosidades son más pronunciadas y rectas. La fosa intercondílea es de contorno rectangular, profunda y amplia, con la línea intercondílea pronunciada, dispuesta en sentido del cóndilo lateral.

\section{DISCUSIÓN}

La ornamentación de los osteodermos es muy importante para identificar los diferentes taxones de Pampatheriidae (Edmund \& Theodor, 1997), al igual que en el resto de los Cingulata. En particular los osteodermos no marginales de los escudos escapular y pélvico, y los osteodermos no marginales de los cinturones móviles son muy útiles al momento de caracterizar una especie.

Los osteodermos no marginales de los escudos escapular y pélvico de Scirrotherium antelucanus sp. nov., a nivel biométrico muestran áreas (sensu Edmund, 1996, Góis et al., 2013) mucho mayores que los homólogos de Scirrotherium hondaensis Edmund \& Theodor, 1997, Vassallia minuta (Moreno \& Mercerat, 1891) y Plaina subintermedia (Rovereto, 1914), (fig. 2); de hecho su menor rango de área $\left(627-1324 \mathrm{~mm}^{2}\right)$ se ubica en el extremo superior del mayor rango de área de S. hondaensis $\left(312-683 \mathrm{~mm}^{2}\right)$. Sin embargo, $S$. hondaensis muestra espesores ligeramente mayores o similares a los de Scirrotherium antelucanus sp. nov. (fig. 4, apéndice 2) y superficies similares o ligeramente mayores a las de Kraglievichia paranense Ameghino, pero con espesores algunos de ellos mucho menores (apéndice 2).

Por otra parte, el área media de Scirrotherium antelucanus sp. nov. en general, es inferior que el área media de Vassallia maxima, pero su espesor medio coincide con algunas muestras de esa especie por lo que sus dimensiones se traslapan parcialmente, siendo ésta especie la que presenta mayor coincidencia biométrica con Scirrotherium antelucanus sp. nov. (fig. 2).

$\mathrm{Si}$ se compara con la nueva especie de Scirrotherium de Argentina y Brasil (Góis et al., 2012), el espesor promedio de Scirrotherium antelucanus sp. nov. tiene áreas o superficies mucho más grandes y espesores conspicuamente más gruesos; además, la ornamentación de la especie argentino-brasileña es notable por la 


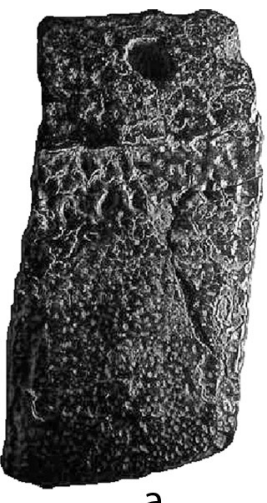

a
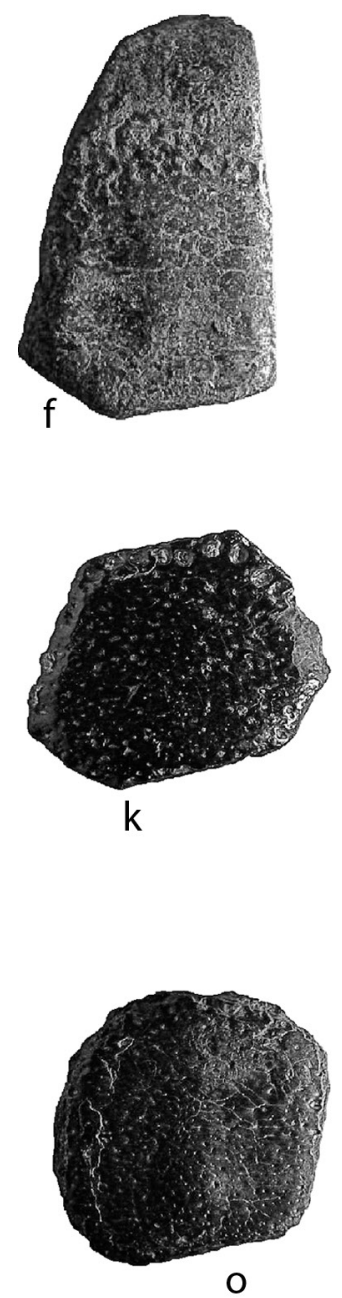

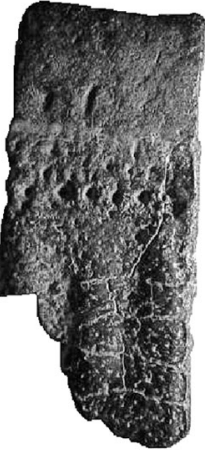

b
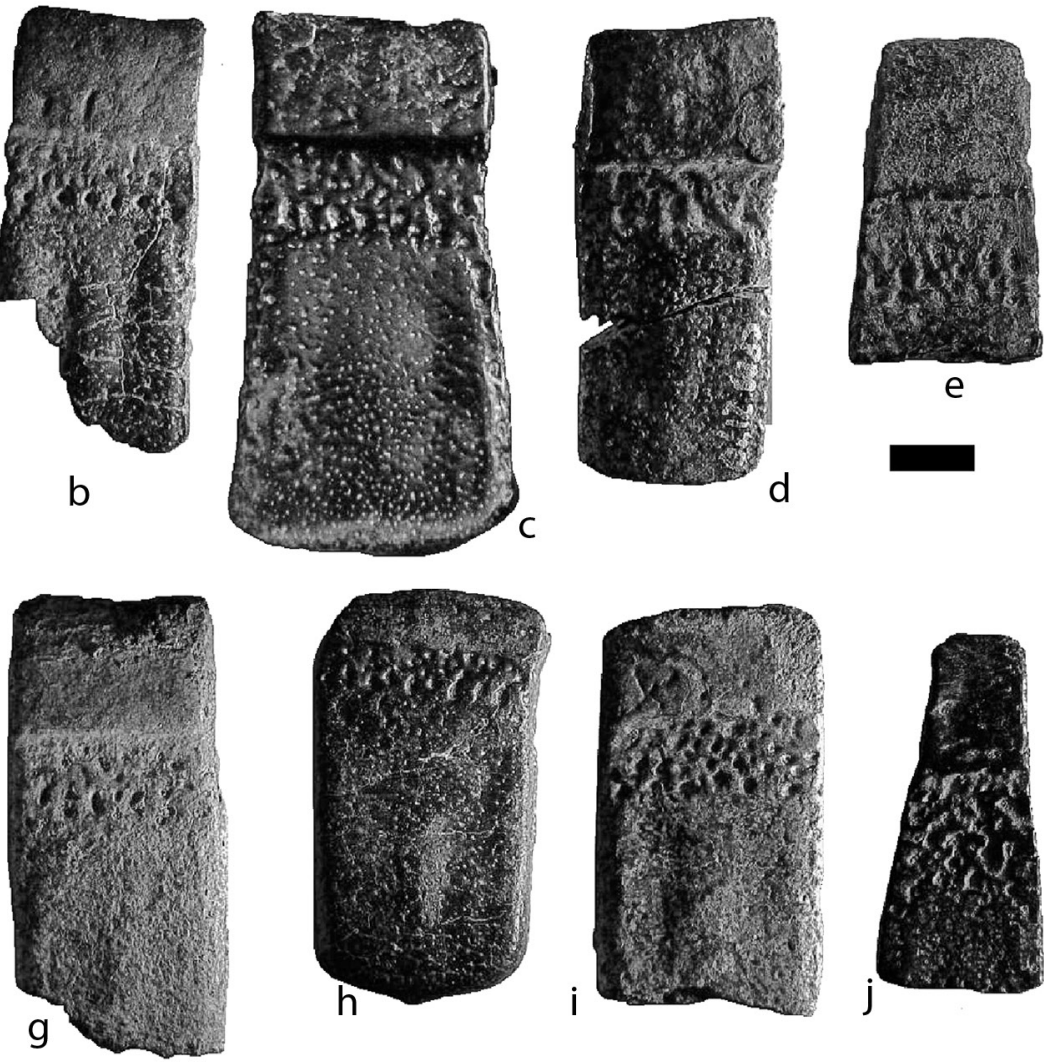

e
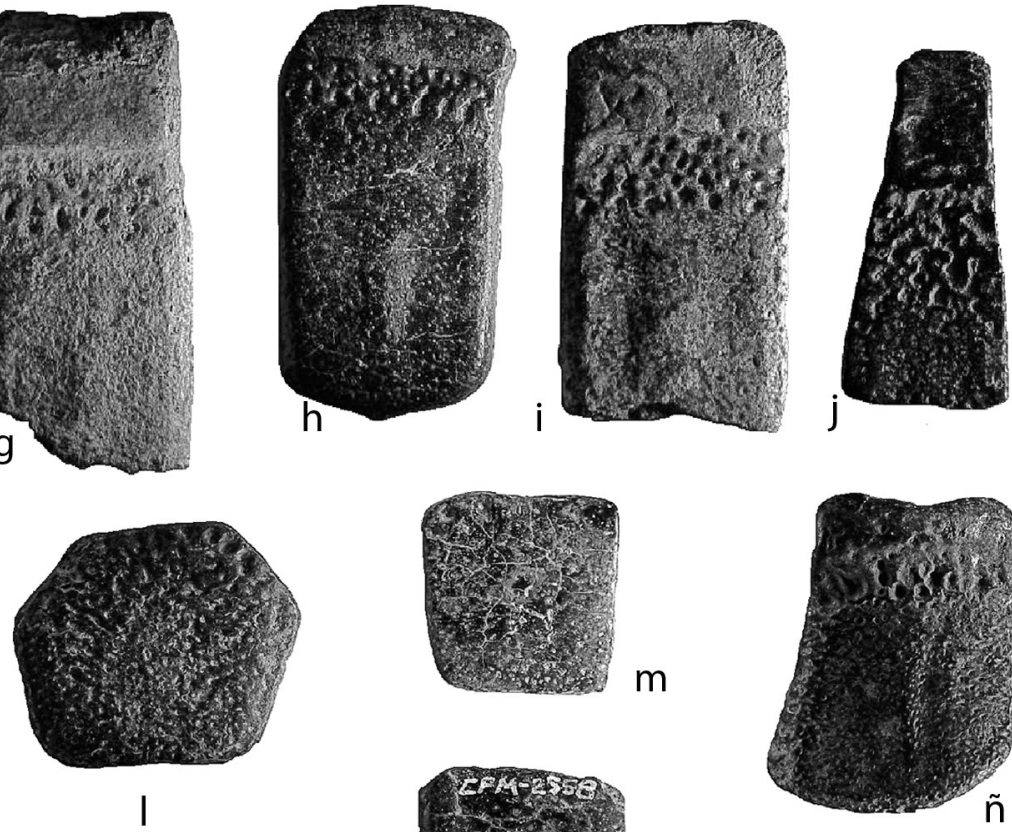

m
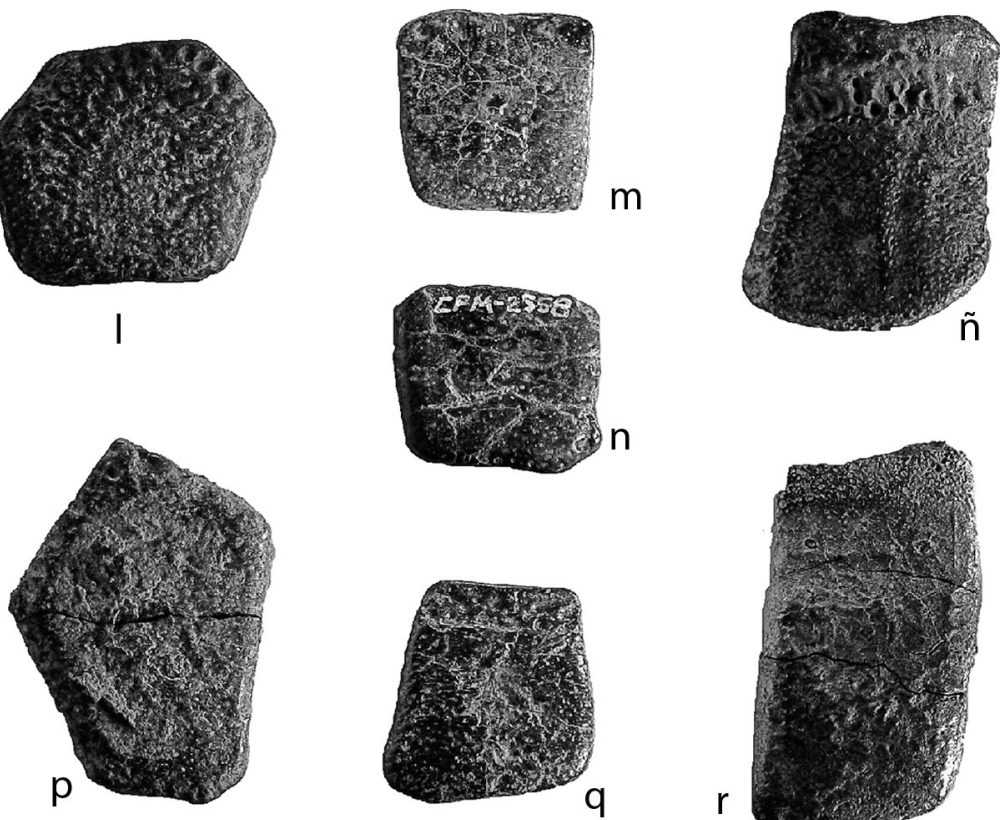

Fig. 6: a-g y j, osteodermos de las bandas móviles; h, osteodermo semi-móvil; k-1 y ñ osteodermos del escudete cefálico; m, n, o y p, osteodermos del escudo escapular y q-r, osteodermos del estuche caudal (escala gráfica $1 \mathrm{~cm}$ ). 
presencia de una elevación central longitudinal angosta, elongada y elevada a manera de carena que recuerda a la especie Kraglievichia paranense, en tanto que la especie costarricense presenta elevaciones centrales

Aparte de V. maxima, otras tres especies de pampaterios caen en el domino biométrico de Scirrotherium antelucanus sp. nov.; Kraglievichia paranense (Ameghino, 1883) con un área media ligeramente menor y un espesor medio ligeramente mayor; y los especímenes más pequeños de las especies pleistocénicas Pampatherium humboldti (Lund, 1839) y Pampatherium typum (Ameghino, 1875), pero como la mayoría de los especímenes de Pampatheriidae Plio-pleistocenos de los género Pampatherium, así como Holmesina presentan osteodermos notablemente más grandes y gruesos con áreas medias en general mucho mayores, por lo que se descarta la inclusión de los especímenes costarricenses dentro de alguno de estos géneros (fig. 3).

Si se compara con el pampaterio mio-pliocénico Plaina intermedia (Ameghino, 1888) esta última especie presenta áreas y espesores mayores, similares a Vassallia maxima. Además, Plaina intermedia (Ameghino, 1888) presenta caracteres muy similares a $V$. maxima de allí que De Iuliis \& Edmund (2002)

En el caso de Plaina brocherense, el material asignado presenta dimensiones notablemente mayores que las de especie Scirrotherium antelucanus (fig. 2); De Iuliis \& Edmund (2002), consideran que Plaina brocherense debe ser referida al género Vassallia ya que los osteodermos son muy similares a Vassallia máxima pero más grandes.

$\mathrm{Si}$ se realizan comparaciones temporales, es posible descartar todas las especies de los géneros Holmesina y Pampatherium, por poseer rangos de distribución temporal restringidos al Plioceno tardío y Pleistoceno. Las únicas coincidencias temporales observables se dan con las especies Vassallia máxima Castellanos, 1946 y Kraglievichia paranense (Ameghino, 1883).

A nivel morfológico, los osteodermos de la especie Kraglievichia paranense (Ameghino, 1883) se diferencian de los de S. antelucanus esp. nov. por tener superficies externas con amplias elevaciones centrales longitudinales, que en el caso de los osteodermos fijos son notablemente sub-circulares y en los osteodermos móviles son sub-rectangulares, en general inflados; en tanto que en $S$. antelucanus esp. nov. son piriformes $\mathrm{y}$ angostos en el caso de los osteodermos fijos y aunque sub-rectangulares en algunos osteodermos móviles, en general son angostos y en todos los casos son bajos. La figuras o elevaciones centrales longitudinales en Kraglievichia paranense (Ameghino, 1883) están bordeadas por profundas y relativamente alargadas depresiones longitudinales, cuyas superficies presentan una conspicua ornamentación constituida por una serie de ranuras someras (cf. Edmund \& Theodor, 1997 y Góis \& Scillato-Yané, 2010; ver fig. 1c de Edmund, 1987 y fig. 7.3 de Candela et al., 2012), todas esas características que son comparables a las del género Holmesina.

Por el contrario las depresiones longitudinales en S. antelucanus esp. nov. son poco profundas $\mathrm{y}$ aunque en algunos casos se observan algunas ranuras que ornamentan su superficie, estás son poco evidentes, más angostas y menos profundas. Por último, el espesor de los osteodermos fijos de Kraglievichia paranense (Ameghino, 1883) poseen una media de $7.3 \mathrm{~mm}$ con un máximo de 11 mm y un mínimo de $6 \mathrm{~mm}$, ello supera notablemente a $S$. antelucanus esp. nov. con una media de $6.17 \mathrm{~mm}$, un máximo de $7.10 \mathrm{~mm}$ y un mínimo de $4.91 \mathrm{~mm}$, marcando una importante diferencia entre dichas especies (ver apéndices 1 y 2).

Al comparar los osteodermos no marginales de los cinturones fijos de Scirrotherium antelucanus sp. nov. con los de Vassallia maxima Castellanos, 1946, se hacen las siguientes diferencias:

La elevación marginal de $S$. antelucanus esp. nov. se diferencia de la elevación homóloga de Vassallia máxima Castellanos,1946 por carecer de rugosidades u ornamentación marcada, adicional a los forámenes.

El sulcus o depresión longitudinal es muy amplio y somero, en forma de herradura en las placas hexagonales, pero es notablemente elongado en los escudos rectangulares producto de la forma perimetral. Comparado con el sulcus de los escudos de $V$. maxima, el sulcus de $S$. antelucanus nov. sp. es mucho más ancho, pero al igual que en 


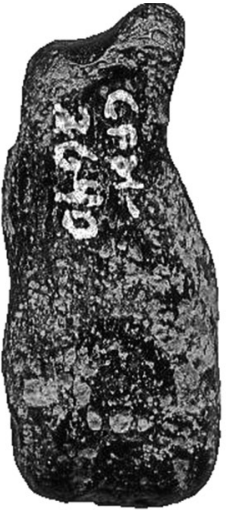

a

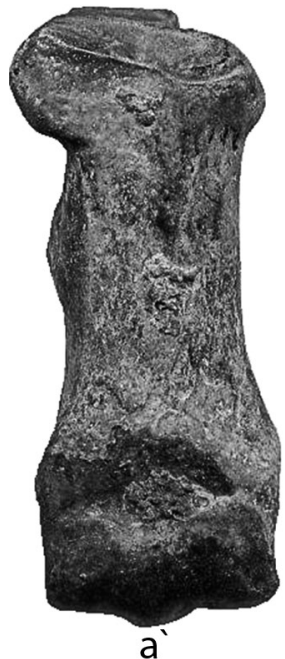

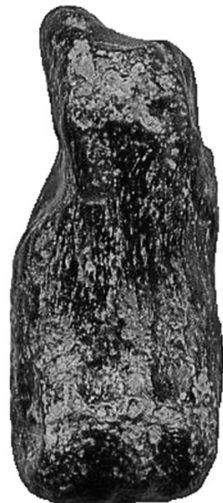

b

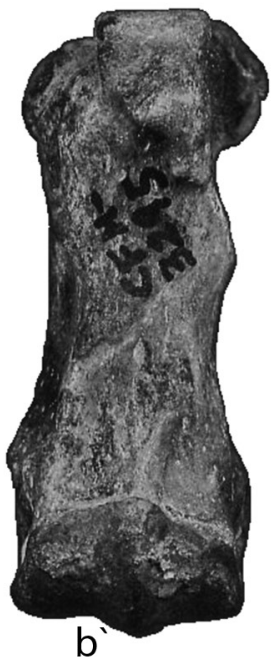

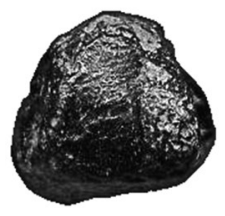

C

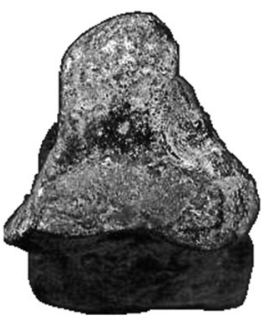

$C^{\prime}$

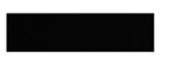

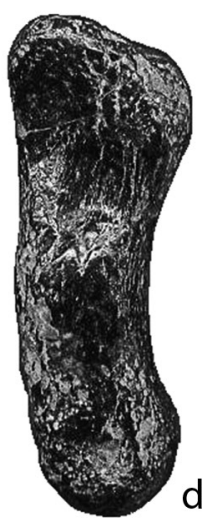
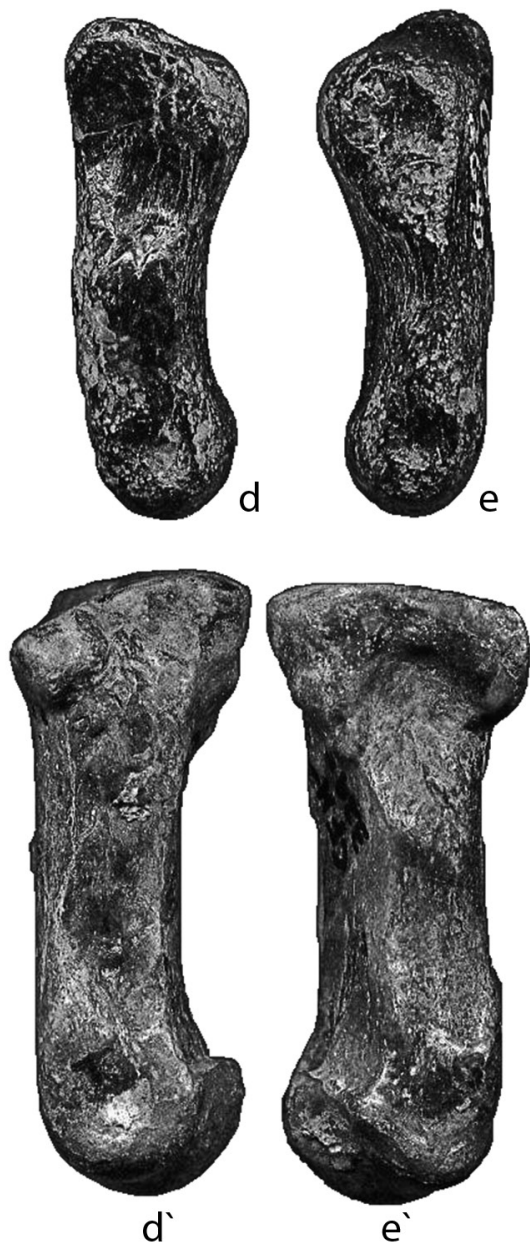

Fig. 7: Elementos post-craneales de Scirrotherium antelucanus, CFM-2640 II metatarso izquierdo en norma a: dorsal, b: plantar, c: proximal, d: medial y e: lateral; CFM- 3295 III metatarso derecho en norma a': dorsal, b': plantar, c': proximal, d': medial y e': lateral (escala gráfica $1 \mathrm{~cm}$ ).

Vassallia maxima Castellanos, 1946 en algunos osteodermos el sulcus se vuelve ligeramente más angosto por un ensanchamiento de la elevación marginal.

La figura central es piriforme, ligeramente elevada, alargada y angosta y se une a la banda submarginal en el extremo posterior. En $V$. maxima la figura central es ancha, baja y de contorno casi ovoide o subrectangular de ángulos redondeados y se proyecta mucho distalmente en tanto que en $S$. antelucanus sp. nov. la figura central no se proyecta más allá de los dos tercios anteriores.
Al comparar los anchos de la elevación central longitudinal (AECL) de los osteodermos escapulares de Scirrotherium antelucanus sp. nov. con los anchos AECL de los osteodermos homólogos de Vassallia minuta, Vassallia maxima y Kraglievichia paranense (ver tabla 3 de Góis et al., en prensa y el apéndice 1), la especie costarricense se caracteriza por tener anchos cuyos rangos mínimo y máximo de $2.26 \mathrm{~mm}$ y 7.33 $\mathrm{mm}$ respectivamente y una media $5.16 \mathrm{~mm}$, son notablemente menores que la media de Vassallia minuta con $7.48 \mathrm{~mm}$, un mínimo de $6.9 \mathrm{~mm}$ y un máximo de $9.5 \mathrm{~mm}$; Vassallia maxima con una 
media de $18.35 \mathrm{~mm}$, un mínimo de $15.5 \mathrm{~mm}$ y un máximo de $21 \mathrm{~mm}$; y Kraglievichia paranense con una media de $11.29 \mathrm{~mm}$, un mínimo de $9 \mathrm{~mm}$ y un máximo de $13 \mathrm{~mm}$.

Por último y de manera adicional a las comparaciones hechas entre los osteodermos no marginales de los cinturones fijos de Scirrotherium antelucanus sp. nov. con los de Scirrotherium hondaensis (v. supra), se hacen las siguientes diferencias: osteodermos más grandes con superficies más suaves, con forámenes más grandes y conspicuos, en mayor número ( 8 a 10 contra 6 a 7 en Scirrotherium hondaensis) y el ancho de la elevación central longitudinal en Scirrotherium hondaensis con una media $6.66 \mathrm{~mm}$ es mayor que la media homóloga de Scirrotherium antelucanus sp. nov. con $5.16 \mathrm{~mm}$.

\section{Distribución paleogeográfica y edad}

El género Scirrotherium, inicialmente se consideró monoespecífico y la especie Scirrotherium hondaensis fue descrito para el Mioceno Medio tardío de la Formación La Venta, Grupo Honda de Colombia por Edmund \& Theodor (1997). Más recientemente, la especie de Scirrotherium carinatum Góis et al., 2013 ha sido registrada en el "Conglomerado Osífero" de la Formación Ituzaingó, provincia de Entre Ríos, asignado al Huayqueriense (Cione et al., 2000; Góis \& Scillato-Yané, 2010) y en las localidades de Punta Delgada y Pico Lobo de la Formación Puerto Madryn en la península de Valdés, provincia de Chubut de edad Huayqueriense [10.8 a 8.7 Ma] (Dozo et al., 2002, 2008, 2010), ambas de Argentina. Esa misma especie nueva se ha registrado en la Formación Solimôes del Mioceno tardío del Estado de Acre en Brasil (Goís et al., 2013). Además, existe un hallazgo no descrito aún de Scirrotherium en el Cenozoico de Venezuela (com. esc. Rincón, 2009).

La localidad fosilífera de San Gerardo de Limoncito en el sur de Costa Rica, corresponde a la ubicación más septentrional del género, aunque falta aún revisar la determinación del Pampatheriidae Plaina hallado en dos localidades de San Miguel Allende en el centro de México y de edad Blancano [3.9 Ma] (Carranza-Castañeda \& Miller, 2004; Carranza-Castañeda, 2005) que aunque no ha sido descrito en detalle, podría tratarse de una forma coevolutiva y eventualmente la más septentrional del género Scirrotherium.

La edad asignada a Scirrotherium antelucanus nov. sp. es Mioceno Superior, Hemphilliano temprano (Hh1-Hh2) con base en la fauna fósil de caballos con la que se encontró asociado, a saber las especies Dinohippus mexicanus (Lance, 1950), Protohippus gidleyi Hulbert, 1988 y Calippus hondurensis (Olson \& McGrew, 1941) (Valerio, 2010; Laurito \& Valerio, 2010) y el Xenarthra, Megalonychidae, Pliometanastes cf. protistus (Laurito \& Valerio, 2012b).

\section{Paleoecología}

El registro del género Scirrotherium en el Mioceno de América del Sur está asociado a faunas de mamíferos terrestres, teleósteos, tiburones y moluscos propios de climas cálidos, húmedos y tropicales (Cione et al., 2005; Dozo et al., 2010). Kay \& Madden (1997) determinaron para la localidad La Venta del Mioceno Medio del centro de Colombia, donde se registra también el género Scirrotherium, un ambiente con predominio de tierras húmedas, bajas y tropicales. Condiciones similares a las de la localidad de San Gerardo de Limoncito, donde la fauna de mamíferos, reptiles y peces permiten reconstruir un ambiente tropical próximo a la costa con sabanas arboladas, vegetación mixta y extensos humedales correspondientes a una zona de vida basal relativamente cálida y húmeda (Valerio, 2010; Laurito \& Valerio, 2010, 2012a).

\section{CONCLUSIONES}

Se describe por primera vez para América Central una nueva especie de Pampatheriidae del género Scirrotherium, Scirrotherium antelucanus procedente del Mioceno tardío del sur de Costa Rica. Con este hallazgo, se amplía la distribución paleogeográfica del género fuera de América del 


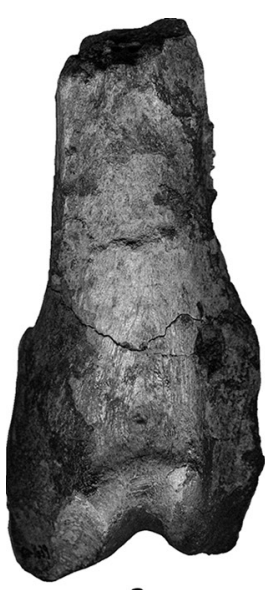

a

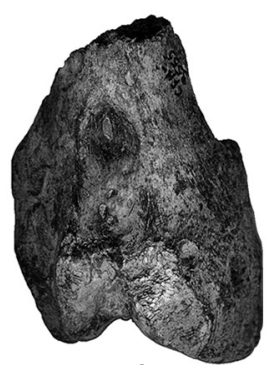

$a^{\prime}$

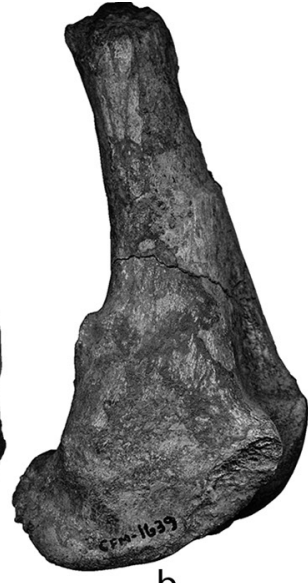

b

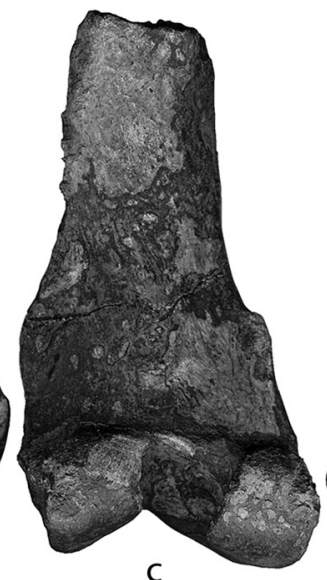

C

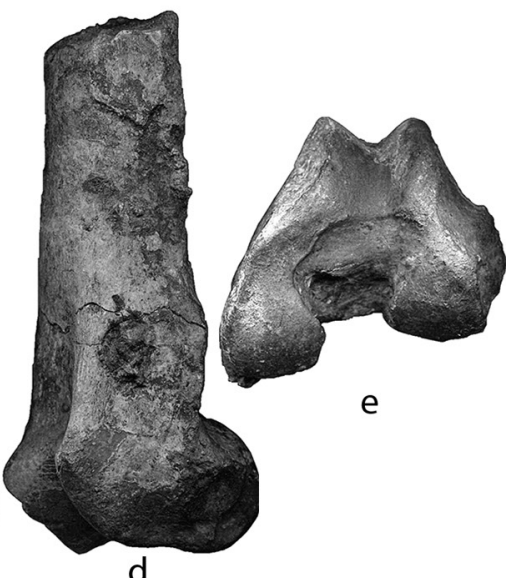

d

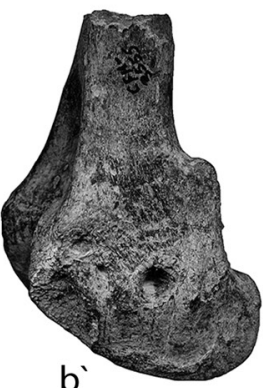

b

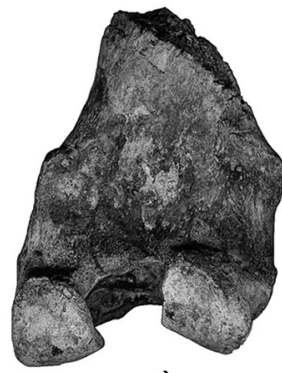

$c^{\prime}$

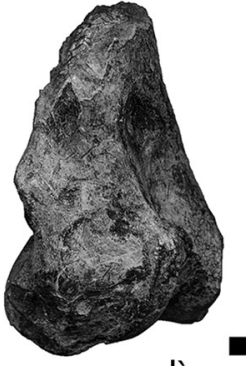

d'

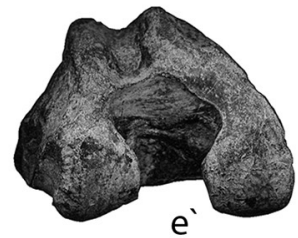

e

Fig. 8: Elementos post-craneales de Scirrotherium antelucanus, fragmentos distales de fémur, de a-e el CFM-1639 fémur derecho y de a'-e' el CFM-3555 fémur izquierdo en vista a, a': anterior; b, b': lateral externa; c. c': posterior; d, d': lateral mediana y e, e': distal (escala gráfica $5 \mathrm{~cm}$ ).

Sur y el conocimiento de los Heraldos suramericanos (sensu Webb, 1976) durante el Pre-GABI.

El arribo de Scirrotherium antelucanus o su ancestro a la porción más meridional del Arco de Islas Costa Rica-Panamá, durante el Mioceno tardío, se ubica en el final del escenario de Syncolisión y al inicio del escenario de Post-colisión del sur de dicho arco con el noroeste de Colombia sensu Coates et al. (2004). Momento en que se conjugaron una fuerte caída del nivel del mar y el levantamiento de Arco de Islas Costa Rica Panamá, lo que le permitió a estos armadillos gigantes junto con otros xenartras saltar de manera efectiva las islas (Laurito \& Valerio, 2012a). Su migración, probablemente se vio favorecida por la continuidad climática entre el norte de América del Sur y el sur de América Central, ya que para el Mioceno tardío y el Plioceno temprano las asocia- ciones paleoflorísticas del sur de América Central sugieren ambientes tropicales cálidos y húmedos, bosques lluviosos de zonas bajas sin evidencia de vegetación de altura, árida o seca (Graham \& Dilcher, 1998), condiciones más afines a las existentes en el noroeste de América del Sur para el Mioceno medio, época en la que se registró en la localidad de La Venta el género Scirrotherium (Kay \& Madden, 1997).

El presente registro de Scirrotherium antelucanus, incrementa la antigüedad del arribo de la familia Pampatheriidae al Hemisferio Norte, en al menos $3 \mathrm{Ma}$ antes del inicio del Gran Intercambio Biótico Americano y es probable que su diversificación en el Hemisferio Norte se diera durante el Plioceno temprano (ver el registro de "Plaina" en el Plioceno temprano del centro de México, cf. Carranza-Castañeda \& Miller, 2004 y Flynn et al., 
2005) y se continuara en el Pleistoceno. Ello cambia la suposición previa que se tenía, de que arribaron en el Plioceno temprano y se diversificaron en el Plioceno tardío (según Marshall et al., 1984; Stehli \& Webb, 1985; Morgan \& Hulbert, 1995; Carranza-Castañeda \& Miller, 2004; Flynn et al., 2005; Scillato-Yané et al., 2005; Carlini \& Zurita, 2010; Woodburne, 2010).

\section{AGRADECIMIENTOS}

Al Dr. Alfredo Carlini quién nos suministró algunas imágenes de Scirrotherium hondaensis y realizó numerosas e importantes observaciones que permitieron la mejora del texto; al Dr. Flávio Goís por la copia de la prueba de impresión de su trabajo sobre la nueva especie de Scirrotherium de América del Sur; ambos del Departamento Científico de Paleontología de Vertebrados del Museo de La Plata, Buenos Aires, Argentina. De igual forma se le agradece a la Dra. María Teresa Dozo del Laboratorio de Paleontología del Centro Nacional Patagónico en Puerto Madryn, Chubut, Argentina, por sus comentarios que facilitaron la determinación del material y la bibliografía brindada.

Nuestro especial reconocimiento al Dr. Ascanio Rincón, del Instituto Venezolano de Investigaciones Científicas (IVIC), Centro de Ecología y Laboratorio de Biología de Organismos en Caracas, Venezuela; quién de manera desinteresada ayudó con el suministro de los datos biométricos comparativos de prácticamente todas las especies de Pampatheriidae conocidas y la inclusión y graficación de los datos costarricenses utilizados en este trabajo.

Por último agradecemos a la profesora de latín, Dra. Flavia M. Siérkovich Bartoli de Morera, ciudad de Palmares, quién nos orientó en la elección del nombre de la especie.

\section{REFERENCIAS}

AGUILAR, D. \& LAURITO, C., 2009: El armadillo gigante (Mammalia, Xenarthra, Pampatheriidae) del río Tomayate,
Blancano tardío-Irvingtoniano temprano, El Salvador, América Central.- Rev. Geol. Amér. Central, 41: 25-35.

CANDELA, A.M., BONINI, R.A. \& NORIEGA, J.I., 2012: First continental vertebrates from the marine Parana' Formation (Late Miocene, Mesopotamia, Argentina): Chronology, biogeography, and paleoenvironments. - Geobios, 45(6): 515-526.

CARLINI, A.A. \& SCILLATO-YANÉ, G.J., 1993: Origin and evolution of "glyptodontoids".J. Vert. Paleont. 13 (3 Suppl.), 28A.

CARLINI, A.A. \& ZURITA, A.E., 2010: An introduction to cingulate evolution and their evolutionary history during the Great American Biotic Interchange: biogeographical clues from Venezuela.- En: SÁNCHEZVILLAGRA, M.R., AGUILERA, O.A. \& CARLINI, A.A. (eds.): Urumaco \& Venezuelan Paleontology. - Indiana University Press, 233-255.

CARRANZA-CASTAÑEDA， O., 2005: Un nuevo inmigrante sudamericano, en el Blancano temprano (Plioceno) del Centro de México.- Geos, 25 (1): 152.

CARRANZA-CASTAÑEDA, O. \& MILLER, W., 2004: Late Tertiary Terrestrial Mammals from Central Mexico and their relationship to South American Inmigrants.- Rev. Brasileira de Paleont. 7(2): 249-261.

CASTELLANOS, A., 1927: Breves notas sobre los clamidoterios.- Publicaciones del Centro de Estudiantes de Ingeniería de Rosario, 1-8.

CASTELLANOS, A., 1937: Anotaciones sobre las líneas filogenéticas de los clamidoterios.Publicaciones de la Facultad de Ciencias, Universidad Nacional del Litoral, Argentina, serie Técnica y Científica, 8: 1-35.

CASTELLANOS, A., 1946: Una nueva especie 
de clamiterio, Vassallia maxima n. sp.Publicaciones del Instituto de Fisiografía y Geología de Rosario, 26: 1-47.

CIONE, A.L., AZPELICUETA, M.M., CASCIOTTA, J.R. \& DOZO, M.T., 2005: Tropical freshwater teleosts from Miocene beds of eastern Patagonia, southern Argentina.- Geobios, 38: 29-42.

CIONE, A.L., AZPELICUETA, M.M., BOND, M., CARLINI, A.A., CASCIOTTA, J.R., COZZUOL, M.A., DE LA FUENTE, M., GASPARINI, Z., GOIN, F.J., NORIEGA, J.I., SCILLATO-YANÉ, G.J., SOIBELZON, L.,TONNI, E.P., VERZI, D.H. \& VUCETICH, M.G., 2000: Miocene vertebrates from Entre Ríos province, eastern Argentina. - En: ACEÑOLAZA, F.G. \& HERBST, R. (eds.): El Neógeno de Argentina. - Serie Correlación Geológica, 14: 191-237.

COATES, A.G., COLLINS, L.S., AUBRY, M. \& BERGGREN, W.A., 2004: The Geology of the Darien, Panama, and the late MiocenePliocene collision of the Panama arc with northwestern South America.- Geol. Soc. Amer. Bull. 116: 1327-1344.

DE IULIIS, G. \& EDMUND, A.G., 2002: Vassallia máxima Castellanos, 1946 (Mammalia: Xenarthra: Pampatheriidae), from Puerta del Corral Quemado (Late Miocene to Early Pliocene), Catamarca Province, Argentina.En: EMRY, R.J. (ed.): Cenozoic Mammals of Land and Sea: Tributes to the Career of Clayton E. Ray.- Smithsonian Contributions to Paleobiol. 93: 49-64.

DOZO, M.T., BOUZA, P., TAMBUSSI, C. \& MASSAFERRO, G., 2008: Vertebrados continentales de edad Huayqueriense (Mioceno Tardío) en Península Valdés (Chubut, Argentina): implicancias biocronológicas, paleoambientales y paleobiogeográficas.- III Congreso Latinoamericano de Paleontología de Vertebrados, Neuquén, Patagonia, Argentina.
DOZO, M.T., MONTI, A., BOUZA, P., VUCETICH, M.G., CIONE, A.L., TONNI, E.P. \& SCILLATO-YANÉ, G.J., 2002: Geología y vertebrados continentales en cercanías de Punta Delgada (Neógeno de Península Valdés, Chubut, Argentina).- En: CABALERI, N., CINGOLANI, C.A., LINARES, E., LÓPEZ DE LUCHI, M.G., OSTERA, H.O. \& PANARELLO, H.O. (eds.): Actas del XV Congreso Geológico Argentino CD-ROM, Artículo No. 118: 1-6.

Dozo, M.T., Bouza, P., Monti, A., Palazzesi, L., Barreda, V., Massaferro, G., Scasso, R.A. \& Tambussi, C.P., 2010: Late Miocene continental biota in Northeastern Patagonia (Península Valdés, Chubut, Argentina).Palaeogeography, Palaeoclimatology, Palaeoecology, 297: 100-109.

EDMUND, A.G., 1985: The Armor of fossil giant Armadillos (Pampatheriidae, Xenarthra, Mammalia).- $\quad$ Pearce-Sellards-Series, Texas Memorial Museum, 40: 1-20.

EDMUND, A.G., 1987: Evolution of the Genus Holmesina (Pampatheriidae, Mammalia) in Florida, with Remarks on Taxonomy and Distribution.- Pearce-Sellards Series, Texas Memorial Museum, 45: 1-20.

EDMUND, A.G., 1996: Areview of the Pleistocene giant Armadillos (Mammalia, Xenarthra, Pampatheriidae). - En: STEWART, K.M. \& SEYMOUR, K.L. (eds), Palaeontology and Palaeoenvironments of Late Cenozoic Mammals: Tributes to the Career of C.S. (Rufus) Churcher.- University of Toronto Press, Toronto, 300-321.

EDMUND, A.G. \& THEODOR, J., 1997: A new giant armadillo. - En: KAY, R.F., CIFELLI, R.L., FLYNN, J.J. \& MADDEN, R.H. (eds.): Vertebrate Paleontology of the Miocene Honda Group, Republic of Colombia.- Smithsonian Inst. Press, Washington, 227-232. 
FLYNN, J.J., KOWALLIS, B.J., NÚÑEZ, C., CARRANZA-CASTAÑEDA, Ó., MILLER, W.E., SWISHER, C.C., III, \& LINDSAY, E.H., 2005: Geochronology of Hemphillian-Blancan aged strata, Guanajuato, Mexico, and implications for timing of the Great American Biotic Interchange.- J. of Geol. 113: 287-307.

GILLETTE, D.D. \& RAY, G.E., 1981: Glyptodonts of North America.- Smithsonian Contributions to Paleobio. 40: 1- 255.

GÓIS, F.L. \& SCILLATO-YANÉ, G.J., 2010: Nueva especie de Scirrotherium (Xenarthra, Cingulata, Pampatheriidae) del "Conglomerado osífero" (Mioceno tardío), base de la Formación Ituzaingó, provincia de Entre Ríos, Argentina.- X Congreso Argentino de Paleontología y Bioestratigrafía y VII Congreso Latinoamericano de Paleontología Resúmenes.

GÓIS, F.L., SCILLATO-YANÉ, G.J., CARLINI, A.A. \& GUILHERME, E., 2013: A new species of Scirrotherium Edmund \& Theodor, 1997 (Xenarthra, Cingulata, Pampatheriidae) from the late Miocene of South America.- Alcheringa 37: 177-188.

GRAHAM, A. \& DILCHER, D.L., 1998: Studies in Neotropical Paleobotany. XII. A Palynoflora from the Pliocene Rio Banano Formation of Costa Rica and the Neogene Vegetation of Mesoamerica.- Amer. J. of Botany, 85(10): 1426-1438.

HOFFSTETTER, R., 1956: Contribution à l'étude des Orophodontoidea Gravigrades cuirassés de la Patagonie.- Annales de Paléontologie, 42: 25-64.

HULBERT, R.C., 1988: Calippus and Protohippus (Mammalia, Perissodactyla, Equidae) from the Miocene (Barstovian-Early Hemphillian) of the Gulf Coastal Plain.Bull. of the Florida State Mus. Biol. Sci. 32(3): 221-340.
HULBERT, C.H. \& MORGAN, G.S., 1993: Quantitative and qualitative evolution in the giant armadillo Holmesina (Edentata: Pampatheriidae) in Florida.En: MARTIN, R.A. \& BARNOSKY, A.D. (eds): Morphological change in Quaternary mammals of North America.- Cambridge University Press, Cambridge, 134-178.

KAY, R.F. \& MADDEN, R.H., 1997: Mammals and rainfall: paleoecology of the middle Miocene at La Venta (Colombia, South America).- J. of Human Evolution, 32: 161-199.

LANCE, J.F., 1950: Paleontología y Estratigrafía del Plioceno de Yepómera, Estado de Chihuahua $1^{\text {a }}$ parte: Equidos, excepto Neohipparion.- Universidad Nacional Autónoma de México, Instituto de Geología, 54: 1-81.

LAURITO, C. \& VALERIO, A., 2008: Primer registro de Pampatheridae en Costa Rica (Formación Curré, Hemphilliano Temprano): la evidencia más temprana del inicio del Intercambio Faunístico entre las Américas vía América Central.- IX Congreso Geológico de América Central. Programas y resúmenes. p. 109.

LAURITO, C. \& VALERIO, A., 2010: Los caballos fósiles de la Formación Curré, cantón de Coto Brus, Costa Rica.- 131 págs. Museo Nacional de Costa Rica, San José.

LAURITO, C. \& VALERIO, A., 2012a: Paleobiogeografía del arribo de mamíferos suramericanos al sur de América Central previo al gran intercambio biótico americano: Un vistazo al GABI en América Central.- Rev. Geológica de América Central, 46: 123-144.

LAURITO, C. \& VALERIO, A., 2012b: Primer registro fósil de Pliometanastes sp. (Mammalia, Xenarthra, Megalonychidae) 
para el Mioceno Superior de Costa Rica, América Central. Una nueva pista en la comprensión del Pre-GABI.- Rev. Geológica de América Central, 47: 95-108.

MARSHALL, L., BERTA, A., HOFFSTETTER, R., PASCUAL, R., REIG, O., BOMBIN, M. \& MONES, A., 1984: Mammals and stratigraphy-geochronology of the continental mammal-bearing Quaternary of South America.- Palaeovertebrata, Mémoire Extraordinaire 1984: 1-76.

MEAD, J., CUBERO, R., VALERIO, A., SWIFT, S., LAURITO, C. \& GÓMEZ, L., 2006: Plio-Pleistocene Crocodylus (Crocodylia) from southwestern Costa Rica.- Studies on Neotropical Fauna and Environment, 41(1): 1-7.

MORGAN, G.S. \& HULBERT, R.C. JR., 1995: Overview of the geology and vertebrate paleontology of the Leisey Shell Pit Local Fauna, Hillsborough Country, Florida.Bull. Florida Mus. Natural History, 37:1-92.

OLSON, E.C. \& MCGREW, P.O., 1941: Mammalian fauna from the Pliocene of Honduras. - Bull. of the Geol. Soc. of Amer. 52: 1219-1244.

RINCÓN, A.D., WHITE, R.S. \& MCDONALD, G., 2008: Late Pleistocene Cingulate (Mammalia: Xenarthra) from Mene de Inciarte, Sierra de Perijá, western Venezuela.J. Vert. Paleont. 28(1): 197-207.
SCILLATO-YANÉ, G.J., CARLINI, A.A., TONNI, E.P. \& NORIEGA, J.I., 2005: Paleobiogeography of the late Pleistocene pampatheres of South America.- J. of South Amer. Earth Sci. 20: 131-138.

STEHLI, F.G. \& WEBB, S.D., 1985: A kaleidoscope of plates, faunal and floral dispersals, and sea level changes. - En STEHLI, F.G. \& WEBB, S.D. (eds.): The Great American Biotic Interchange.- Capítulo 1: 3-16. Plenum Press, Nueva York-Londres.

VALERIO, A.L., 2010: Paleontología, bioestratigrafía y paleoecología de los caballos fósiles de la Formación Curré en el Cantón de Coto Brus, Costa Rica (análisis basado en material dental).- 353 págs. Univ. de Costa Rica, San José [Tesis Lic.].

WEBB, S.D., 1976: Mammalian faunal dynamics of the Great American Interchange.Paleobiology, 2: 216-234.

WOODBURNE, M.O., 1969: A late Pleistocene occurrence of the collared peccary, Dicotyles tajacu, in Guatemala.- J. of Mammalogy, 50:121-125.

WOODBURNE, M.O., 2010: The Great American Biotic Interchange: dispersals, tectonics, climate, sea level and holding pens.- J. of Mammalian Evolution, 17: 245-264. 
Apéndice 1

Cuadro de datos biométricos de los osteodermos fijos no marginales de Scirrotherium antelucanus especie nueva

\begin{tabular}{|c|c|c|c|c|c|}
\hline Placa & Largo (mm) & Ancho (mm) & Grosor $(\mathrm{mm})$ & Área $\left(\mathrm{mm}^{2}\right)$ & Figura \\
\hline CFM-3113 & 28,57 & 21,97 & 4,91 & 627,68 & No ilustrado \\
\hline CFM-1205 & 30,74 & 24,81 & 5,60 & 762,66 & No ilustrado \\
\hline CFM-1677 & 33,01 & 26,34 & 6,36 & 869,48 & No ilustrado \\
\hline CFM-1158 & 30,62 & 28,91 & 6,65 & 885,22 & Fig. 3o \\
\hline CFM-3545 & 36,36 & 24,44 & 5,58 & 888,64 & Fig. 31 \\
\hline CFM-1580 & 33,16 & 27,51 & 6,55 & 912,23 & Fig. 3n \\
\hline CFM-1975 & 34,15 & 27,12 & 6,25 & 926,15 & No ilustrado \\
\hline CFM-1582 & 34,04 & 27,53 & 6,00 & 937,12 & Fig. 3ñ \\
\hline CFM-1850 & 36,15 & 26,19 & 5,00 & 946,77 & Fig. $3 \mathrm{k}$ \\
\hline CFM-2157 & 35,72 & 26,52 & 6,98 & 947,29 & Fig. $3 \mathrm{j}$ \\
\hline CFM-1581 & 34,70 & 27,34 & 6,18 & 948,70 & Fig. $3 f$ \\
\hline CFM-3378 & 33,56 & 28,65 & 5,60 & 961,49 & No ilustrado \\
\hline CFM-1203 & 34,30 & 28,21 & 6,34 & 967,60 & Fig. $3 \mathrm{c}$ \\
\hline CFM-2569 & 37,81 & 26,65 & 6,37 & 1007,64 & No ilustrado \\
\hline CFM-2828 & 34,26 & 29,59 & 5,70 & 1013,75 & Fig. $3 \mathrm{~h}$ \\
\hline CFM-1583 & 36,66 & 27,88 & 6,08 & 1022,08 & No ilustrado \\
\hline CFM-1821 & 37,38 & 27,59 & 6,88 & 1031,31 & Fig. $3 \mathrm{i}$ \\
\hline CFM-3111 & 38,76 & 27,02 & 6,77 & 1047,30 & Fig, $3 g$ \\
\hline CFM-1963 & 37,09 & 29,33 & 6,30 & 1087,85 & No ilustrado \\
\hline CFM-2965 & 37,62 & 28,99 & 7,10 & 1090,60 & Fig. 3e \\
\hline CFM-3544 & 41,01 & 29,09 & 6,94 & 1192,98 & No ilustrado \\
\hline CFM-2829 & 46,12 & 26,08 & 6,08 & 1202,81 & No ilustrado \\
\hline CFM-3540 & 40,56 & 30,64 & 6,40 & 1242,76 & Fig. 3a \\
\hline CFM-2559 & 40,80 & 32,02 & 6,05 & 1306,42 & Fig, 3b \\
\hline CFM-3297 & 40,93 & 32,37 & 5,70 & 1324,90 & No ilustrado \\
\hline Sumatoria & 904,08 & 692,79 & 154,37 & 25151,45 & \\
\hline Desviación & 0,5769 & & & & \\
\hline Media $=$ & 36,16 & 27,71 & 6,17 & 1006,06 & \\
\hline
\end{tabular}


Apéndice 2

Tabla de datos biométricos de los osteodermos fijos no marginales de Scirrotherium hondaensis y Scirrotherium especie nueva para Brasil y Argentina (sensu Edmund \& Theodor, 1997 y Góis et al., 2013).

\begin{tabular}{|c|c|c|c|c|c|}
\hline & Espécimen & Largo $(\mathrm{mm})$ & Ancho (mm) & Grosor $(\mathrm{mm})$ & Área $\left(\mathrm{mm}^{2}\right)$ \\
\hline Scirrotherium Ar-Br & MLP41-XII-13-905 & 33,5 & 25 & 6 & 837,5 \\
\hline Scirrotherium Ar-Br & MLP41-XII-13-914A & 26,5 & 22,1 & 5,9 & 585,65 \\
\hline Scirrotherium Ar-Br & MLP41-XII-13-914B & 29,9 & 17,3 & 4,9 & 517,27 \\
\hline Scirrotherium Ar-Br & MLP41-XII-13-924 & 26,5 & 20,5 & 4,5 & 543,25 \\
\hline Scirrotherium Ar-Br & MLP52-X-1-36A & 29,5 & 19,9 & 5 & 587,05 \\
\hline Scirrotherium Ar-Br & MLP52-X-1-36B & 27 & 20,5 & 4,9 & 553,5 \\
\hline Scirrotherium Ar-Br & MLP69-IX-8-13AC & 24,5 & 20 & 4,5 & 490 \\
\hline Scirrotherium Ar-Br & MLP69-IX-8-13AF & 20,5 & 19,9 & 5 & 407,95 \\
\hline Scirrotherium Ar-Br & MLP69-IX-8-13AG & 24,5 & 20 & 4,5 & 490 \\
\hline Scirrotherium Ar-Br & MLP69-IX-8-13AH & 29,5 & 25,5 & 5 & 752,25 \\
\hline Scirrotherium Ar-Br & MLP69-IX-8-13AI & 24 & 16,5 & 4,5 & 396 \\
\hline Scirrotherium Ar-Br & MLP69-IX-8-13AJ & 30 & 26,1 & 4,5 & 783 \\
\hline Scirrotherium Ar-Br & MLP69-IX-8-13AK & 23 & 17 & 4,2 & 391 \\
\hline Scirrotherium Ar-Br & MLP69-IX-8-13AL & 22 & 19,9 & 5 & 437,8 \\
\hline Scirrotherium $\mathrm{Ar}-\mathrm{Br}$ & MLP69-IX-8-13AM & 25 & 17,5 & 4,1 & 437,5 \\
\hline Scirrotherium Ar-Br & MLP69-IX-8-13AÑ & 20,9 & 22 & 4,1 & 459,8 \\
\hline Scirrotherium Ar-Br & MLP69-IX-8-13AO & 24 & 18 & 5 & 432 \\
\hline Scirrotherium Ar-Br & MLP70-XII-29-1 & 24 & 19,2 & 5,5 & 460,8 \\
\hline S. hondaensis & UCMP 37924 & 26,9 & 22,3 & 6,9 & 599,87 \\
\hline S. hondaensis & UCMP 37924 & 24,5 & 27,9 & 6,8 & 683,55 \\
\hline S. hondaensis & UCMP 40056 & 25,2 & 21,2 & 7 & 534,24 \\
\hline S. hondaensis & UCMP 88381 & 16 & 19,5 & 7 & 312 \\
\hline S. hondaensis & UCMP 88381 & 17,5 & 21,5 & 5 & 376,25 \\
\hline S. hondaensis & UCMP 88381 & 22 & 17,5 & 6,5 & 385 \\
\hline
\end{tabular}

